\title{
WhatsApp in a clinical setting: The good, the bad and the law
}

\author{
C J Opperman, ${ }^{1} \mathrm{MB}$ ChB, BScHons (Microbiol); M Janse van Vuuren, ${ }^{2}$ LLB, MSc (Physiol Sci) \\ ${ }^{1}$ Department of Pathology, Faculty of Health Sciences, University of Cape Town, South Africa \\ ${ }^{2}$ Chennells Albertyn Attorneys, Stellenbosch, South Africa
}

Corresponding author: C J Opperman (stefanopperman1@gmail.com)

\begin{abstract}
The mounting complexity of patient data, with the increasing burden of patient numbers, demands efficient communication among a multidisciplinary team of healthcare providers, to ensure a smooth-running hospital. In the daily function of a microbiology laboratory, information must be shared rapidly between doctors of various hospitals and disciplines. A doctor in the clinical setting may feel the need to be at different places at the same time in order to function efficiently. Therefore, the argument for using instant messaging to alleviate these pressures is compelling. The concern, however, is the high incidence of identifiable and sensitive data being exchanged. This article focuses on the justification for using WhatsApp Messenger in the clinical setting, with an emphasis on bioethics and the current legal framework in South Africa.
\end{abstract}

S Afr J Bioethics Law 2018;11(2):102-103. DOI:10.7196/SAJBL.2018.v11i2.643

Giordano et al. ${ }^{[1]}$ in 2017 published a systematic review article on WhatsApp Messenger (WhatsApp) as an adjunctive tool for telemedicine. Telemedicine is defined as the use of electronic communication technologies to provide healthcare support and information between a client and professional with expertise in the relevant field when separated by distance. They found a small number of eligible studies, which did not address ethics or security issues. It was concluded from their review that WhatsApp is a reliable, costeffective, quick and user-friendly tool that improves patient-related awareness and communication within the clinical health sector.

\section{The good}

In the microbiology laboratory, it is crucial to share pertinent results with doctors to facilitate problem identification and ensure immediate management of patients, which would directly affect the outcome of treatments. WhatsApp has become central to the flow of information from the laboratory to the general practitioner, which in turn enhances the feedback process. Its feasibility and acceptability extend into the primary care setting, in that WhatsApp is not only used to inform colleagues of results, but also provides a platform to integrate theory and clinical practice for students. Furthermore, it clarifies aspects of uncertainty in patient bedside management with real-time conversations between junior and senior physicians. ${ }^{[2]}$ In a microbiology laboratory specifically, WhatsApp enhances professional networking and a culture of advice-sharing between pathologists and community service doctors in the peripheral hospitals.

\section{The bad}

It is easy to imagine how WhatsApp can bring worlds closer, with information literally being at a doctor's fingertips, but are we paying a moral and ethical price for this? A recent study at University Hospital Limerick in Ireland revealed that $97 \%$ of the surveyed doctors shared sensitive information over instant messenger without patient consent, despite $68 \%$ of them being concerned about the distribution of this information. ${ }^{[3]}$ The neglect of the doctor-patient confidentiality clause remains a worry with the use of WhatsApp in a clinical setting. The authority bestowed upon a clinician, within the patient-doctor relationship, places the patient in a vulnerable position. The patient's information should be guarded in confidence, within reasonable limits, to maintain the trust of the public in the medical profession and its traditional virtues.

To the best of our knowledge, after a thorough investigation of the current available literature, no litigation pertaining to the use of WhatsApp has been brought before a South African (SA) court or ethical review board. The idea of legal action arising from social media is still new in SA law, with the first case being that of Isparta v Richter in 2013, which resulted in the North Gauteng High Court awarding damages to the plaintiff, after suing the defendants for defamation arising from the posting of certain comments on the defendant's Facebook wall. ${ }^{[4]}$

The security of sensitive data containing patient-identifiable information is another concern. A lost phone, or the unauthorised use thereof, can lead to a breach in security by an unknown and unidentifiable assailant. The Limerick study showed that $30 \%$ of the interns interviewed had lost their phones in the last year, and $5 \%$ in the last week. ${ }^{[3]}$ It should be noted that WhatsApp uses end-to-end encryption as a method to protect data, which even prevents the parent company from accessing the information, as well as double password protection, which includes phone and WhatsApp lock. ${ }^{[3]} \mathrm{A}$ software solution that ensures password protection is essential to maintain accountability among users; however, even with the aforementioned security protocols, healthcare providers must still exercise reasonable caution when posting patient-identifiable results.

Further technical hurdles that may be encountered by healthcare providers using WhatsApp include, but are not limited to, internet connectivity problems, variability in image quality, communications not always being included in a patient's medical record, frequent 
interruptions on ward rounds and discussions that cannot easily be printed ${ }^{[5]}$ Another problem with WhatsApp is that of primary responsibility and ownership. Feedback on active WhatsApp groups can be absent when clinicians do not respond. This leaves an open loop of communication, resulting in a lack of primary responsibility and ownership of results. Furthermore, non-adherence to professional boundaries by WhatsApp users, especially among medical students and junior doctors, is troublesome. These may include discriminatory language, profanity and photographs of patients without their consent. ${ }^{[6]}$ Education around professionalism and the use of social media should therefore start at medical school orientation, and continue until graduation.

\section{And the law}

Section 14 of the Bill of Rights in the SA Constitution ${ }^{[7]}$ entrenches a patient's right to privacy. While this right might not be absolute, the limitation thereof should be rational and justifiable in terms of section 36 of the Constitution. Since the adoption of the final Constitution in 1996 , numerous pieces of legislation have been promulgated to give effect to a person's right to privacy. ${ }^{[7]}$

Section 14(1) of the National Health Act No. 61 of 2003 (NHA) stipulates that all information concerning a patient, including information relating to his/her health status, treatment or stay in a health establishment, is confidential. Subsection (2) further prescribes that no person may disclose any information contemplated in subsection (1) unless the patient consents to such disclosure in writing, or when required to do so by a court order or by law, or if nondisclosure of such information may lead to a serious public threat. ${ }^{[8]}$

The position regarding the requirements for the disclosure of a patient's information is further articulated in the Ethical Rules of Conduct for Practitioners Registered under the Health Professions Act No. 56 of 1974, as published in the Government Gazette Notice R717 in August 2006. ${ }^{[9]}$ According to rule 13, a healthcare provider may only divulge information regarding a patient, verbally or in writing, either with the express consent of said patient, the written consent of his/her guardian in the event of a minor patient under the age of 12 years, or in the event of a deceased patient, the written consent of the deceased's next of kin, or the executor of such deceased patient's estate.

It is important to note that section 15 of the NHA states that a healthcare provider may disclose a patient's information to any other person, healthcare provider or health establishment as is necessary for any legitimate purpose within the ordinary course and scope of his/her duties where such a disclosure is in the interest of the patient. As the Act does not specify in which form, or through what medium, such a disclosure should be made, it can be argued that the use of WhatsApp to communicate such a disclosure would follow the Act, granted that all other requirements of the relevant section are complied with. ${ }^{[8]}$

In the absence of informed consent by a patient, or where the requirements of section 15 of the NHA have not been met, the use of WhatsApp to share medical information holds a risk of legal consequences, especially if it results in harm to the patient as a result of an unlawful disclosure. Even though the abovementioned legislation imposes restrictions on the use and sharing of a patient's information, the decision is very rarely made to share information on WhatsApp without the patient's consent or legal justification.
Ethical principles guide physicians towards a higher moral responsibility to serve the public. These principles are enforced by the Health Professions Council of SA (HPCSA) and underpinned by the Constitution, the statutes which give effect to the Constitution as well as relevant jurisprudence. The HPCSA has ethical guidelines to guide healthcare providers on the use of telemedicine, but not social media. However, a practical and ethical guide for medical students and doctors has been published online by the South African Medical Association (SAMA). ${ }^{[4]}$ This includes recommendations on how to avoid the minefield of ethical and legal issues encountered by healthcare providers using social media. It should be noted that there is a fundamental difference between social media platforms such as Facebook or Twitter, as mentioned in the SAMA guidelines, and confidential exchange of information by WhatsApp. Doctors ought to remember that not abiding by ethical guidelines may threaten their reputation and practice.

\section{Conclusion}

Professional councils such as the HPCSA should direct healthcare providers on good ethical practice in the use of social media, but medical schools must also teach these principles from an early stage. The development of sophisticated software technology turns WhatsApp into an attractive and potentially secure communication tool, but with great power comes great responsibility. If information is shared among healthcare providers on WhatsApp without the consent of a patient, it must be justifiable in the eyes of the law, in the patient's best interest and able to withstand moral judgment if questioned on professional boards.

Acknowledgements. We would like to acknowledge Prof. Mark Nicol, head of the Division of Medical Microbiology, Department of Pathology, Faculty of Health Sciences, University of Cape Town, for providing insight into the article.

Author contributions. Equal contributions.

Funding. None.

Conflicts of interest. None.

1. Giordano V, Koch MD, Godoy-Santos A, et al. WhatsApp messenger as an adjunctive tool for telemedicine: An overview. Interact J Med Res 2017;6(2):1-8. https://doi.org/10.2196/ijmr.6214

2. Raiman L, Antbring R, Mahmood A. WhatsApp messenger as a tool to supplement medical education for medical students on clinical attachment. BMC Med Educ 2017;17(7):1-9. https://doi.org/10.1186/s12909-017-0855-x

3. O'Sullivan DM, O'Sullivan E, O'Connor M, et al. WhatsApp Doc? BMJ Innov 2017;3:38-239. https://doi.org/10.1136/bmjinnov-2017-000239

4. Kubheka B. Ethical and legal perspectives on use of social media by health professionals in South Africa. S Afr Med J 2017;107(5)386-389. https://doi.org/10.7196/ SAMJ.2017.v107i5.12047

5. Boulos MNK, Giustini DM, Wheeler S. Instagram and WhatsApp in health and healthcare: An overview. Future Internet 2016;8(3):1-14. https://doi.org/10.3390/ fi8030037

6. Nikiphorou E, Berenbaum F. Patient-physician interaction on social media: The physician's point of view. EMJ Innov 2018:2(1):40-42.

7. Constitution of the Republic of South Africa, 1996. Chapter 2: Bill of Rights.

8. South Africa. National Health Act No. 61 of 2003.

9. South Africa. Ethical Rules of Conduct for Practitioners Registered under the Health Professions Act No. 56 of 1974, ad Rule 13. Government Gazette 29079, 4 August 2006.

Accepted 16 July 2018. 